\title{
PERCEPCIÓN DE LA FUNCIÓN PROFESIONAL DELNUTRICIONISTA POR PARTE DE LOS USUARIOS DE ATENCIÓN PRIMARIA
}

\section{PERCEPTION OF THE NUTRITIONIST'S PROFESSIONAL FUNCTION FROM PRIMARY CARE USERS}

\author{
Patricio Oliva M. (1), Kristian Buhring B. (2), Sofia Godoy T. (3), María Bustos T. (3) \\ (1) Dirección de Ciencias Básicas, Metodología e Investigación. \\ Facultad de Odontología, Universidad del Desarrollo. Concepción, Chile. \\ (2) Departamento de Salud Pública. Facultad de Medicina. \\ Universidad Católica de la Santísima Concepción. Concepción, Chile. \\ (3) Facultad de Medicina. Universidad Católica de la Santísima Concepción. Concepción, Chile.
}

\begin{abstract}
Research to assess the role or to describe the functions of the health personnel from the perspective of the patients is not common in the health area; however, its study allows a valid feedback in defining both a professional profile and setting targets. The objective of this investigation was to describe the most important dimensions that external users consider when defining "socially and subjectively" the professional nutritionist. This was a phenomenological qualitative study using semi-structured interviews with 28 users of Conception health centers. The sample was qualitative and operates through saturation point, semantic analysis was structural. As a result we obtained the description of the role of the nutritionist classified in the dimensions in which patients recognize the work, confirming some of its functions and discovering the importance of other ancillary and secondary consideration. Key words: perception, nutritionists, primary care.
\end{abstract}

Este trabajo fue recibido el 31 de Marzo de 2009 y aceptado para ser publicado el 1 de Marzo de 2010.

\section{INTRODUCCIÓN}

El concepto de cultura dentro del campo de la salud constituye el eje neurálgico en el análisis de los factores sociales involucrados en cualquier proceso de salud y enfermedad (1), de ella derivan elementos constituyentes de la realidad social que explican la interacción entre los individuos y el sistema sanitaria como tal. Por lo cual la definición de las acciones profesionales en salud puede ser comprendida de mejor forma si se conocen las distintas visiones de los actores involucrados, siendo los pacientes un elemento fundamental, siendo sus creencias en las distintas manifestaciones de la dicotomía saludenfermedad (2). Con ello se favorece una retroalimentación, de carácter subjetivo, pero concordante con los principios cualitativos de la investigación.

La percepción de las funciones por parte de los pacientes de los profesionales de salud conllevan por otra parte consecuencias tan básicas como la elección del profesional al momento de la consulta, o bien influir en los niveles de adherencia a tratamientos prescritos.

La descripción de la función del profesional nutricionista no ha sido caracterizado desde el punto de vista operativo por parte de los pacientes hasta la fecha, por lo cual, en el presente estudio se indaga de forma cualitativa, por medio del análisis de discurso, donde lo importante no es la cuantificación sino el análisis y la interpretación del lenguaje (3), debido a que la metodología cualitativa permite conocer la percepción, que los sujetos tienen de sus vivencias cotidianas, de una manera que los diseños cuantitativos no lo permiten (4), de las cuales se obtienen categorías que describen las funciones específicas del nutricionista, enmarcado dentro del sentido de las representaciones sociales en el lenguaje de los individuos $\mathrm{y}$ en base a las situaciones que estos se encuentran (5).

\section{SUJETOS Y MÉTODO}

Se realizó una investigación cualitativa, de carácter descriptiva fenomenológica (6), especialmente los dis- 
cursos y los significados que las personas les otorgan (7). Se escogió este diseño por la flexibilidad y capacidad de adaptarse a las condiciones muestrales que el estudio posee. La investigación posee una muestra no probabilística de caso tipo, constituidos por 14 hombres y 14 mujeres, pertenecientes a los Centros de Salud de Atención Primaria Dr. Víctor Manuel Fernández, Santa Sabina y O'Higgins, todos de la comuna de Concepción (con un total de 7 nutricionistas en conjunto), con un punto de corte de entrevistas regido por el punto de saturación (8), lo que significa que la investigación se finalizó cuando las respuestas de los entrevistados comienzan a repetirse en todas sus categorías. El periodo de recolección de la información duró 3 meses del año 2009. Los criterios de selección se remitieron a adultos, mayores de 18 años, que eran pacientes de servicios de atención primaria, de ambos sexos, en condiciones de responder las preguntas y que hayan sido atendidos o estaban actualmente en tratamiento con un nutricionista.

A la muestra se le aplicó una entrevista Semiestructurada (9), instrumento que consiste en una pauta de preguntas (la construcción de la pauta deriva del cuadro lógico) que derivan del problema investigativo, que le da la estructura, pero que además posee la capacidad de permitir contrapreguntar o reorientar las preguntas, configurando el procedimiento metodológico correcto para el tipo de información que se necesita encontrar (10). Posee 15 preguntas, previamente piloteadas, construidas a partir de una información bibliográfica apropiada, con un lenguaje acorde a la muestra estudiada, aplicada en lugares previamente habilitados en los respectivos consultorios.

Se realizó el análisis del contenido de las entrevistas de tipo semántico estructural, que evalúa la comprensión del sentido que tiene para los actores un discurso determinado dentro de contextos sociales específicos. Este sentido el análisis alude a los significados del discurso que un sujeto produce de acuerdo a su contexto y modelo cultural (11). El análisis de los datos implicó: 1) codificar las transcripciones de las entrevistas para categorización y organización del texto en unidades conceptuales más abordables; 2) agrupar códigos por medio de la comparación de los distintos significados obtenidos; 3) generar redes de relación entre familias semánticas para descubrir las dimensiones conceptuales que arrojan.

\section{RESULTADOS}

Derivado del discurso de los pacientes se obtiene una descripción de cómo ellos describen las funciones del profesional nutricionista y en paralelo valorarlo y catalogarlo dentro del equipo de salud. Por lo cual, los resultados pueden agruparse en dos grandes áreas:

a) la visión de la interacción profesional - paciente, que se describe en tres dimensiones específicas:

I) Evaluación individual del estado de salud del paciente: caracterizado por la retroalimentación comunicativa entre la información que el nutricionista puede obtener desde el paciente y que le permite configurar procedimientos de intervención adecuados para cada realidad. Dentro de este contexto, caracterizado por la entrega de pautas de alimentación, necesarias para mejorar o complementar tratamientos de patologías, por lo cual la función del nutricionista se describe por los pacientes como paralelo al tratamiento médico: "El nutricionista es importante porqué trata las enfermedades desde la alimentación, y eso hace más completo todo y uno sana más luego" (Entrevistado ${ }^{\circ} 2$ del Cesfam 1 ).

II) Consejería de las estrategias alimentarias correctas para una adecuada alimentación: caracterizado por la retroalimentación que ocurre cuando el nutricionista entrega información científica de manera accesible a la comprensión del paciente. Por lo cual, la educación cumple dentro del discurso de los individuos las siguientes características: a) Identificación de las problemáticas sociales que condicionan el problema de malnutrición, b) adecuar una rutina alimentaria particular para cada realidad, c) educar y socializar al individuo para que dicho cambio alimentario sea efectivo, incorporándose dentro de la cotidianeidad del individuo: "Es importante porque nos enseñan a medirnos con las comidas, le dan visión de lo que uno puedo comer, le ayudan a formar buenos hábitos alimentarios." (Entrevistado nº del Cesfam n ${ }^{\circ} 1$ ), "El (nutricionista) pregunta mucho lo que pasa en la casa, así puede entenderme que mi problema de gordura es más complicado de lo que parece" (Entrevistado $n^{\circ} 2$ del Cesfam $n^{\circ} 2$ ).

III) Motivación para conseguir una alta adherencia dietoterapeútica por parte de los pacientes: es identificada por éstos como el último eslabón de la atención nutricional, y se caracteriza por evidenciar científicamente las consecuencias que no seguir las recomendaciones nutricionales conllevaría, "siempre me dice lo complicado que me puedo poner si sigo haciendo lo mismo" (Entrevistado $n^{\circ} 6$ del Cesfam no 1 ). También se indica opciones nutricionales que el usuario puede seguir, de acuerdo a la realidad social de cada individuo: "Es fundamental porque si él no me dijera que es lo que tengo malo, yo no sabría que tratamiento seguir". (Entrevistado $\mathrm{n}^{\circ} 13$ del Cesfam $\mathrm{n}^{\circ} 2$ ).

b) Al describir comparativamente las funciones de nutricionista y del resto del profesional de salud, surgen las siguientes categorías en el discurso:

Se describen en el discurso las funciones del nu- 
tricionista comparándolo con otros profesionales de la salud, específicamente en los siguientes puntos: a) llevar un control exhaustivo de los condicionantes psicosociales que determinan las patologías de la cual surgen las consultas, "siempre ven como el lado psicológico de uno, y eso le hace sentir bien a uno" (Entrevistado n ${ }^{\circ} 4$ Cesfam $\left.n^{\circ} 1\right)$. b) Enseñar sobre la correcta manera de alimentarse, lo que implica una comunicación indagativa de los determinantes sociales que pueden estar influyendo en el proceso de malnutrición. Lo anterior conlleva a expresiones tales como: "Las funciones que debe realizar es ayudarnos a mantener nuestro peso adecuado, con dietas y alimentos". (Entrevistado ${ }^{\circ} 10$ del Cesfam $\mathrm{n}^{\circ} 2$ ), o también, "El nutricionista es para que lleve el control de una persona que sea diabética o enferma, entonces para que lleve el control de la alimentación." (Entrevistado $n^{\circ} 8$ del Cesfam $n^{\circ} 2$ ), lo que en definitiva se "definiría como el profesional preocupado de la salud física del paciente." (Entrevistado $\mathrm{n}^{\circ} 9$ del Cesfam $\mathrm{n}^{\circ} 2$ ).

La percepción del nutricionista dentro del equipo de salud, de acuerdo con lo expresado por los pacientes implica a manera de resumen los siguientes puntos:

a) el paciente percibe al nutricionista como un profesional cercano, debido al carácter de la interacción que ocurre en las educaciones.

b) el paciente compara los procedimientos terapéuticos del equipo de salud, estableciendo que el nutricionista posee una comunicación más fluida debido a que sus procedimientos son exclusivamente dietéticos, en contraste con el farmacológico de otros profesionales.

c) el paciente califica al profesional nutricionista como el único indicado para la modificación de dietas, validado por el conocimiento adquirido.

\section{DISCUSIÓN Y CONCLUSIÓN}

En la literatura se menciona que las funciones de los profesionales integrantes del equipo de salud son diferenciadas (12), siendo los nutricionistas considerados como profesionales importantes para el equipo de salud(13). En consecuencia, la presente investigación ha intentado describir la percepción social de la función profesional del nutricionista, basado en las descripciones de las acciones que realiza y de la interacción subsecuente con los pacientes a los cuales atiende en los Centros de Salud de la comuna de Concepción evaluando sus principales características observadas en la atención al público.

En el Centro de Salud, sus funciones son descritas tanto en el papel que cumple en el tratamiento de la malnutrición como en actividades educativas, donde se encuentran principalmente las charlas educativas y las visitas domiciliarias.
Elementos anexos surgen en el discurso que complementan una visión favorable a la adherencia a dietoterapias; según los entrevistados es la empatía que muestra el profesional nutricionista en su consulta, es un individuo acogedor que entregue un buen trato y la disponibilidad de escuchar siempre al usuario externo, especialmente porque los problemas alimentarios están asociados a condiciones psicológicas o sociales particulares, en la cual los elementos emocionales juegan un papel fundamental.

Para que el tratamiento nutricional sea efectivo, según los usuarios externos, es necesario que el paciente confíe en el profesional nutricionista como un profesional completo, capacitado para entregar un buen tratamiento y de calidad, que cuyas intervenciones serán efectivas. La confianza que entrega el nutricionista a los usuarios es respaldada por los años de estudio universitario que lo avalan como profesional.

El Colegio de Nutricionistas de Chile, define al nutricionista como un profesional universitario capacitado para comprender las condicionantes del problema alimentario nutricional. Capaz de comprometer su actuar en la prevención y darle solución a los problemas nutricionales, cuya función principal es ayudar a los pacientes (14). Sin embargo, la definición que tienen los pacientes del profesional nutricionista se basa en la importancia que tiene dentro del Centro de Salud, ya que cumple varias funciones que son de utilidad en el tratamiento integral de la enfermedad. Se concluye que en comparación con la función teóricamente establecida existe semejanza, ya que a pesar de que los usuarios externos no conocen todas las funciones que debe realizar el nutricionista se concentran en las que observan y que les resulta de mayor importancia, ya que influyen directamente en la evolución del tratamiento de su patología.

\section{RESUMEN}

Las investigaciones que evalúan el rol o describen funciones del personal de salud desde la visión de los pacientes no son comunes en el área sanitaria, sin embargo su estudio permite una retroalimentación válida al momento de definir tanto un perfil profesional como el establecimiento de metas para éstos. El objetivo de la investigación fue describir las dimensiones más importantes que los usuarios externos consideran al momento de definir "social e intersubjetivamente" al profesional nutricionista. Se efectuó un estudio cualitativo fenomenológico, mediante entrevistas semi-estructuradas a 28 usuarios de centros de salud de Concepción, muestralmente se operó cualitativamente mediante punto de saturación, su análisis es semántico estructural. Se obtuvo como resultado la descripción de la función del 
nutricionista clasificado en las dimensiones que los pacientes reconocen en su quehacer profesional, ratificando algunas de sus funciones y descubriendo la importancia de otras consideradas anexas y secundarias.

Palabras clave: percepción, nutricionistas, atención primaria.

Dirigir la correspondencia a:

Profesor

Patricio Oliva Mella

Dirección de Ciencias Básicas,

Metodología e Investigación.

Facultad de Odontología.

Universidad del Desarrollo.

Concepción.

Barros Arana 1735 Concepción

Fono fax $56-41-2268501$

E-mail:patriciooliva@udd.cl

\section{BIBLIOGRAFÍA}

1. Ana M Alarcón, Aldo Vidal, Jaime Neira Rozas. Salud intercultural: elementos para la construcción de sus bases conceptuales. Rev Méd Chile 2003; 131: 1064

2. Pasten F. Elaboración de diagnósticos sociales con la utilización de técnicas cualitativas de investigación. Universidad de Valparaíso. Facultad de Derecho y Ciencias Sociales. Escuela de Servicio Social. Editorial Edelva. 2000, pp 18.

3. Conde Fernando, Pérez Andrés Cristina. La Investigación Cualitativa En Salud Pública. Rev Esp Salud Pública 1995; 69: 146.

4. Pérez C. ¿Deben estar las técnicas de consenso incluidas entre las técnicas de investigación cualitativa?. Rev Española Salud Pública 2000; 74 (4). 319-321.

5. Álvarez JL. Cómo hacer investigación cualitativa. Fundamentos y metodología. Editorial Paidós Educador. 2005. pp. 24.

6. Schutz, Alfred. El problema de la realidad social. Amorrotu editores. Argentina. 1995.p. 115.

7. Black N.Why we need qualitative research.J Epidemiol Community Health 1994;48:425-6.

8. Patton MQ.'Designing Qualitative Studies", Purposeful Sampling. en Qualitative Evaluation and Research Methods 1990. Cap.5: pp 169 -186.

9. Remy, J. "Métodos de análisis de contenido de Sociología". Bruselas, Publicación de Universidad Sain Louis; 1991.

10. Valles Miguel "Técnicas de Investigación social”. Reflexión Metodológica y Práctica Profesional. ED. Síntesis Sociológica 1993:97

11. Berger P. Luckmann T. La construcción social de la realidad. Argentina. Amorrotu editores; 2001. pp 101.

12. Dytz JLG, Benzoni SAG, Payno SM. O trabalho multiprofissional na assistência à criança hospitalizada: uma prática fragmentada ou integrada? Acta Pul Enfermagem1997; 10 (1):74-85.

13. Minayo MCS. O. desafío do conhecimento: pesquisa qualitativa em saúde. 7ed. Rio de Janeiro (RJ): Abrasco. 2000.

14. Da Costa S. R.; Trevizan M. Los roles profesionales de un equipo de salud: la visión de sus integrantes. Rev Latino-Americana Enfermagem 2007; 15(1): 106-112. 